\title{
Gestational assessment assessed
}

\author{
Unni Wariyar, Win Tin, Edmund Hey
}

\begin{abstract}
Aims-To review the accuracy with which obstetric information on gestation is recorded in the neonatal records; and the reliability of the methods used for assessing gestational age after birth.

Methods-Service information on all babies born in 1989, and research information on all babies of $<32$ weeks gestation born in the Northern Region in 1990-91, were reviewed to determine the accuracy with which antenatally collected information had been recorded in the neonatal records after birth. A prospective study was also mounted to assess how reliably paediatric staff could assess the gestational age of babies born to mothers with certain obstetric dates under service conditions. Paediatric residents looked at 347 babies of $>32$ weeks gestation, and senior staff looked at 105 babies of $<30$ weeks gestation.
\end{abstract}

Results-The best techniques for estimating gestation immediately after birth were only half as accurate $(95 \% \mathrm{CI} \pm 17$ days) as estimates based on antenatal ultrasound at 15-19 weeks gestation. Assessments that relied on the tone, posture, and appearance of the baby at birth in those of $<32$ weeks gestation were less reliable than assessments based on a retrospective review of when various reflex responses first appeared. They also tended to overestimate true gestation. Antenatal information of high quality was ignored, and arithmetic and transcription errors were introduced during the transfer of antenatal information into over $10 \%$ of postnatal records.

Conclusions-Current ultrasound techniques for "dating" pregnancy antenatally are better than any of the methods of postnatal assessment. Given the reliability of the antenatal information now available, it is regrettable that so many inaccuracies have been allowed to creep into the routine computation and recording of gestation at birth.

(Arch Dis Child 1997;77:F216-F220)

Keywords: gestational assessment; records; antenatal information

It is now 30 years since studies first started describing ways in which paediatricians could assess the gestational maturity of the newborn baby. ${ }^{2}$ These studies were stimulated by a growing awareness that gestation was as important as weight in determining the hazards faced by the baby during and immediately after birth. ${ }^{3}$ Reports also started to appear describing the problems associated with being "light for dates"4 and, because a significant minority of mothers had unreliable or uncertain menstrual dates, simple cotside techniques were soon developed for assessing the maturity of these babies. The approach most frequently adopted used the tone, posture, ${ }^{5}$ and physical appearance ${ }^{1}$ of the baby to develop a composite "score" that could then be used to assess the child's likely gestational age in the period immediately after birth. ${ }^{6}$ The alternative approach, which assessed when various primitive reflexes first became detectable after birth in very preterm babies, ${ }^{7}$ received relatively little subsequent investigation.

This research was designed to assess the usefulness of these two different approaches in babies born more than 10 weeks early, and to compare their accuracy, under service conditions, with that achieved by routine antenatal ultrasound scanning. It was also designed to test, in the same way, the reliability of the three most widely used scoring systems for assessing tone and appearance in babies of 32-42 weeks gestation. The opportunity was also taken to look at the accuracy with which available information on gestation was being recorded.

\section{Methods}

Four separate studies are reported. The first was designed to test how accurately paediatric house staff assessed gestation in babies of 32 or more weeks gestational age using the Dubowitz score $^{6}$ and the shorter Ballard ${ }^{8}$ and Parkin ${ }^{9}$ scoring systems about one month after they took up their appointments, after one half day's training session. Three hundred and forty seven babies were studied, including 142 of less than 37 weeks gestation. Most were assessed by three different doctors. Women were only accepted as having certain dates for this study if they were sure of the date of their last menstrual period, had previously had a regular 26-30 day cycle, had not delivered a baby or used an oral contraceptive in the six months before conception, and had had an ultrasound assessment of biparietal diameter at 15-19 weeks that was compatible with the menstrual dates. These unadjusted menstrual dates were then used for comparative purposes.

A second study was set up to assess four different techniques of assessing the gestational age of very preterm babies. Between 1991 and 1993, a total of 105 babies of less than 30 weeks (210 days) gestation born to mothers with certain menstrual dates at the Princess Mary Maternity Hospital in Newcastle were examined by one of five experienced clinicians, using the assessment techniques described by 


\section{Ballard Score (1979)}

Dubowitz Score (1970)

Parkin Score (1976)

Parkin Score

(no half score allowed) at $\leq 19$ weeks
Obstetric ultrasound

as

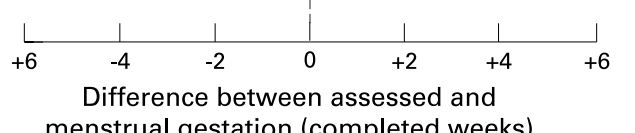

menstrual gestation (completed weeks)

Figure 1 Accuracy of various techniques for assessing gestational age in 347 babies of 32-42 weeks of gestation at birth with certain maternal menstrual dates: bar shows the range of dates within which $95 \%$ of all estimates fell

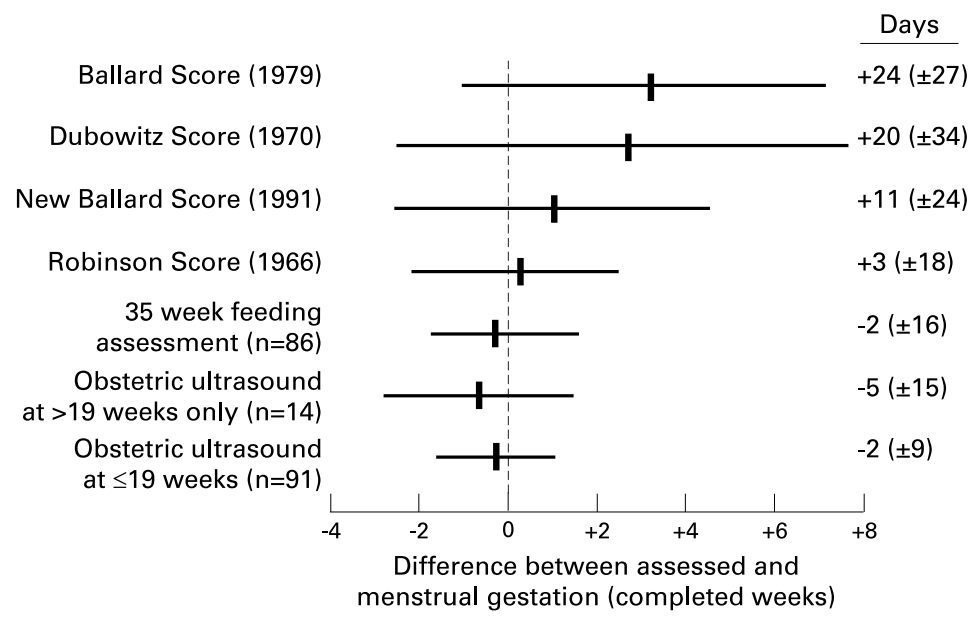

Figure 2 Accuracy of various techniques for assessing gestational age in 105 babies of 29 weeks of gestation or less at birth with certain maternal menstrual dates. Feeding assessment was limited to babies who were no longer oxygen dependent

Robinson, ${ }^{7}$ Dubowitz, ${ }^{6}$ and Ballard. ${ }^{10}$ Criteria for judging the reliability of the menstrual dates were the same as those used for the first study.

A separate study looked at the accuracy with which gestation was recorded at delivery in all the 776 babies enrolled into the controlled trial of early prophylactic fresh frozen plasma, gelatin, or glucose in the Northern Region in 1990-92 (NNNI trial). ${ }^{11}$ Babies were eligible for inclusion in this trial if they were thought to be less than 32 weeks (224 days) gestation at birth. Recruitment had to be registered by telephone within two hours of birth, and staff had to give the trial randomisation centre information regarding gestational age (in completed weeks) in connection with the minimisation process. At a later stage, but before discharge, the local coordinating consultant was required to abstract the obstetric case notes and to record, among other things, the expected date of delivery the obstetricians were using for management purposes (and its certainty) at the time of delivery. During the final data validating process, the case notes of some of the children cared for in each of the trial centres (including each of the nine centres inside or outside the region that provided referral facili- ties) were examined, to eliminate potential data coding errors.

A fourth study looked at the consistency of the computer assembled information on gestation collected in 1989 by the Standard Maternity Information System, on all pregnancies in the Northern Region ${ }^{12}$ before incorporation into the national Hospital Episode System (Maternity and Birth) returns. ${ }^{13}$ This system, now largely abandoned in favour of the minimum Körner data set, recorded the expected date of delivery (based on the best evidence available to the obstetrician before delivery) and the actual date of delivery, as well as the gestational age (in completed weeks) assigned to the baby by the maternity staff after delivery.

In each of these studies gestation was recorded in days, or completed weeks, from the first day of the mother's last menstrual period, the term "postconceptional age" being used to describe the combination of gestational and postnatal age.

\section{Results}

POST DELIVERY ASSESSMENT: BABIES OF 32-42 WEEKS

Three methods of assessing gestational age from the tone, posture, and physical appearance of the newborn baby in the first 2 days of life were compared (fig 1). The standard Ballard score, based on 13 criteria, seemed to be as reliable as the Dubowitz score, based on 21 criteria, but the performance of the Ballard score was actually enhanced by the omission of the six criteria reflecting tone and posture. The Parkin score, based on just four physical criteria, proved as adequate as the shortened Ballard score, but only when staff were encouraged to award half scores for criteria of transitional appearance (a detail not appreciated by some who have doubted the accuracy of this system). ${ }^{14}$

\section{POST DELIVERY ASSESSMENT: BABIES OF < 30} WEEKS

The three methods advocated for use in babies of less than 32 weeks gestation generated estimates with wide confidence intervals (fig 2). More importantly, all three methods systematically overestimated true gestational age, although this was less true of the New Ballard score ${ }^{10}$ than it was of the score originally described in $1979 .^{\circ}$

The alternative approach, documenting when various primitive reflexes first appeared in the weeks after birth, ${ }^{7}$ performed well in experienced hands (fig 2), but only provided a final composite assessment of the child's probable gestational age at birth when the baby was nearly ready for discharge home from hospital. It did not work as well in the hands of less experienced staff. An assessment based on the simple assumption that most preterm babies will have developed a sufficiently well coordinated suck and swallow reflex to take all their feeds by bottle at a postconceptional age of 35 weeks, unless they are dyspnoeic or oxygen dependent, worked equally well. However, none of the paediatric approaches had confidence 


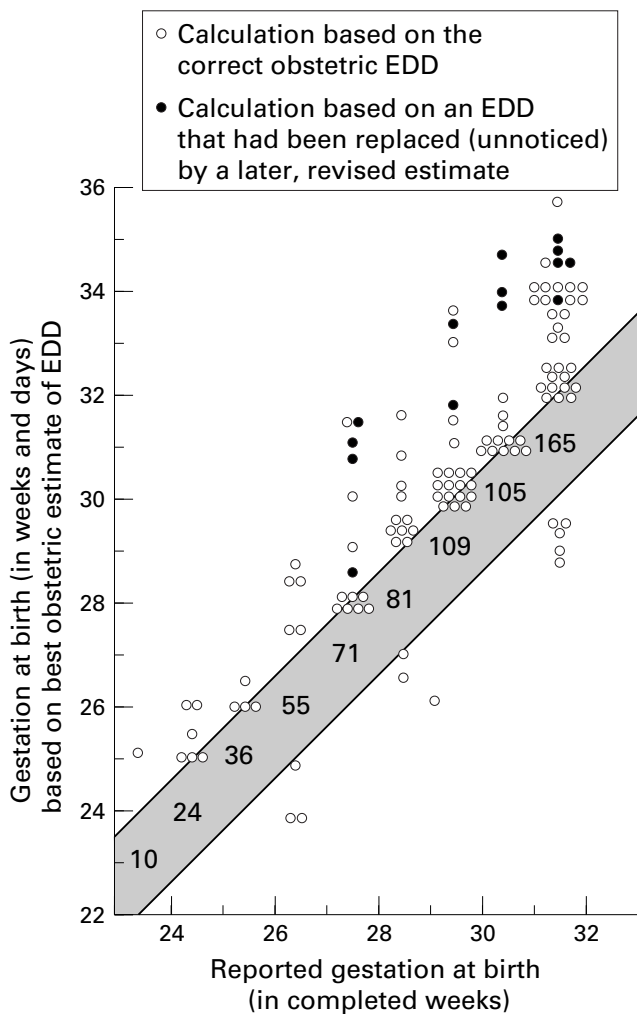

Figure 3 Gestation (in completed weeks), recorded at trial entry on the basis of the obstetric information obtained by neonatal staff at delivery, and gestation (in completed weeks and days), subsequently calculated from the expected date of delivery (EDD), recorded in the obstetric notes.

intervals as narrow as those achieved by antenatal fetal biparietal diameter measurement, especially when this was undertaken at 15-19 weeks (fig 2).

BABIES OF < 32 WEEKS RECRUITED TO THE NNNI TRIAL

Figure 3 shows how gestation, recorded on the basis of the obstetric information obtained by the neonatal staff before trial entry at two hours, correlated with "true" gestational age subsequently calculated from the obstetrician's best estimate of the expected date of delivery. Staff were aware of the importance of reporting this information accurately. Despite this, stated gestation was wrong by at least one week in $15 \%(120 / 776)$ of all trial entrants, and the errors were not random- $92 \%$ of errors involved an underreporting of gestation at birth. The problem seemed to be as common in babies born eight weeks early as in babies born twice as early as this, and errors were found among the returns submitted by every unit in the region.

Later retrospective examination of the case notes showed that the gestation assigned to a baby by the paediatrician after delivery was usually the same as that assigned to a pregnancy by the obstetric or midwifery staff at the time of the mother's admission for care. If a mother was first admitted with toxaemia or threatened labour but only delivered 10 days later, it was not uncommon for her to be still recorded as having delivered at the gestation reached on the day of admission. On other occasions it was less clear why the obstetric staff had unintentionally exaggerated the immaturity of the fetus at the time of the mother's admission: it was almost as if a subconscious decision was being taken to ensure that the mother did not become overoptimistic regarding the pregnancy's likely outcome. In a small number of cases the mother's expected date of delivery had been altered as a result of antenatal ultrasound assessment, but this change was not noticed when the mother was subsequently admitted for delivery, and in every case where this adjustment was overlooked, adjustment made the pregnancy more advanced than was originally thought.

STANDARD MATERNITY INFORMATION SYSTEM RECORDS

A similar analysis was undertaken of all the 33750 computed maternity records of mothers with certain dates delivering in the Northern Region in 1989. Eleven per cent of all the babies thought, on this basis, to have been born before 37 weeks were assigned a different gestation by the maternity staff after delivery, and in $87 \%$ of cases this gestation was less than that calculated on the basis of antenatal dating. The number of term babies recorded as born at 40 weeks gestation was almost twice as high as seemed justified by antenatal information (there being an apparent dearth of deliveries at 38,39, and 41 weeks of gestation).

\section{Discussion}

Before 1960, by international agreement, babies were classified as premature if they weighed $2.5 \mathrm{~kg}$ or less at birth. It was only some years later that it eventually became standard practice to classify babies by their gestation as well as by their weight. ${ }^{15} 16$ The realisation that

\section{Key messages}

- The best techniques for estimating gestation immediately after birth are only half as accurate (95\% CI \pm 17 days) as estimates based on antenatal ultrasound

- Such estimates are even less accurate than this in babies of less than 30 weeks gestation

- Documenting when various reflexes appear can provide a better retrospective estimate of gestation in the preterm baby than methods based on posture, tone and physical appearance

- The transfer of obstetric information relating to gestation into the neonatal record is frequently faulty; such records were in error by $>$ one week in $15 \%$ of preterm babies entered into one recent multi-centre research study, and $92 \%$ of the errors involved an under recording of gestation

- More care should be taken over collecting obstetric information relating to gestation at birth, given its potential value 
gestation was at least as informative as weight gained general acceptance at this time partly because of the growing realisation that the incidence of respiratory distress due to surfactant deficiency varied inversely with gestation at birth, an association which is not only true in preterm babies, ${ }^{17}$ but is also seen in babies of 37 and 38 weeks gestation delivered by elective section before the onset of labour. ${ }^{18} 19$

It soon became clear that other neonatal problems, such as patent ductus, intraventricular haemorrhage, and retinopathy of prematurity, are also influenced by gestation rather than weight at birth (symptoms of any severity being extremely rare in babies of more than 31 weeks gestation), and that the ability to suck and swallow matures quite decisively over quite a short period (fig 2) at a postconceptional age of 34-35 weeks. ${ }^{20}$ Both mortality and morbidity correlate with gestation better then weight in very preterm babies. ${ }^{21}$ Despite this, gestational age is still relatively seldom used when analysing information on morbidity and mortality. One reason for this has been the reliability and universality of birthweight information, and the relative unreliability of much information relating to gestation. It was for precisely this reason that paediatricians started to research ways of estimating gestation age after birth.

Most of the information used when developing the early scoring systems for paediatric assessment of gestation was obtained from relatively mature babies: only two of the babies studied by Dubowitz et $a l,{ }^{6}$ and seven of the babies originally studied by Ballard et $a l,{ }^{8}$ were less than 29 weeks gestation at delivery. Despite this, both scoring systems were widely used to assess less mature babies, and it took many years for the errors inherent in this approach to become apparent. ${ }^{22-26}$ More recently the Ballard score has been reviewed to confirm that the score is not influenced by racial factors, ${ }^{26} 27$ and revised to make it more reliable in very preterm babies. ${ }^{10}$ The evidence reported here suggests that this revision has been only partially successful: the confidence limits associated with any estimate of gestation remain extremely wide, ${ }^{28}$ and the score still tends systematically to overestimate true gestation by at least one week (fig 2), as others have also reported. ${ }^{29}$ The physical components of the score also seem to be more useful than those that rely on tone and posture. ${ }^{26} 29$

Other approaches to the assessment in babies of less than 30 weeks gestation, using serial assessment to determine when various reflexes first become elicitable, have seldom been used, despite having first been described 30 years ago, ${ }^{7}$ having been endorsed by Serfontein and Jaroszewicz ${ }^{30}$ and Parkin, ${ }^{9}$ and advocated in Roberton's Textbook of Neonatology. ${ }^{31}$ The data published here show that such techniques achieve, in experienced hands, a more precise and unbiased estimate of gestation than any technique based on the physical appearance of the baby immediately after birth. Interestingly, however, an assessment that is based merely on the age at which the baby first develops a reliable and coordi- nated suck and swallow reflex, and an ability to bottle feed satisfactorily, seems to pinpoint gestation with equal accuracy in babies who are not dyspnoeic or oxygen dependent. However, none of these techniques estimates gestation as accurately as a well timed antenatal ultrasound measurement of biparietal diameter (figs 1 and 2). Such an assessment can have $95 \%$ confidence intervals of less than \pm 7 days, even under service conditions. ${ }^{32}$

It has been known for some years that paediatric assessment could result in gestation being overestimated in the preterm baby, but the finding that the simple transcription of information from the obstetric to the paediatric records at birth could result in gestation being underestimated was more unexpected. Examination showed that while the structured neonatal case record asked for the gestation of the baby to be written down at birth, in many hospitals, it did not ask for the mother's expected date of delivery to be recorded (or the precision attached to that estimate). Where this information was requested, the date inserted occasionally differed from the working estimate that the obstetricians were using, especially where this had been modified by some days, or sometimes by several weeks, as a result of ultrasound examination. Four cases were found where twins delivering within minutes of each other were assigned gestations that differed by a week or more (the sicker twin being allotted the lower gestation in each case). In eight other cases gestation had been revised by one to two weeks after paediatric assessment, even though this estimate is only accurate to \pm 15 days (95\% CI) at best, despite the fact that the menstrual dates appeared reliable and had been validated by early ultrasound examination. Fifteen cases were found during the case note review where the gestation quoted in the discharge letter in weeks did not agree with that recorded in the neonatal notes, and five cases were found where different gestational ages were quoted in different parts of the same discharge summary. Three babies became two weeks less mature during the course of a one hour journey to a referral centre after delivery because the accompanying letter contained errors. Most of these changes resulted in a downward revision of gestation (fig 3).

Although antenatal information on the most likely date of delivery is now documented during each Confidential Enquiry into Stillbirth or Death, comparable denominator information is not, unfortunately, obtainable from any of the current national maternity data retrieval systems. However, this should not deter clinicians from collecting such data for their own use locally. It will certainly be needed if obstetricians are to have reliable information with which to assess the prognosis for very preterm babies before delivery. ${ }^{21}$ Similarly, if paediatricians are to use gestation rather than birthweight (or some other "risk" score ${ }^{33}{ }^{34}$ ) when comparing survival rates, they will need to eliminate the bias that currently occurs in recording gestation at birth. To conclude, 
birthweight was, for many years, the one piece of information used to determine management and to assess prognosis after birth, because it was the only thing that could be documented accurately (although the accuracy of these weights can be exaggerated ${ }^{35}$ ). Clinicians in all countries where there is universal access to early antenatal care can now collect gestational information of comparable accuracy, and there is evidence that such information is even more informative than birthweight in guiding management. Unlike birthweight, this information is also available to the clinician before delivery. Given the reliability of the information now available, and its potential management value, it is regrettable that so many errors have been allowed to creep into its actual computation and recording at delivery.

The late Professor JM Parkin was responsible for setting up the first of these four studies, and for motivating the resident staf under his supervision to make the observations reported here. $\mathrm{He}$ did not live long enough, unfortunately, to analyse the results.

1 Farr V, Mitchell RG, Neligan GA, Parkin JM. The definition of some external characteristics used in the assessment of gestational age. Dev Med Child Neurol 1966;8:507-11.

2 Farr V, Kerridge DF, Mitchell RG. The value of some external characteristics in the assessment of gestational age at birth. Dev Med Child Neurol 1966;8:657-60.

3 Dunn PM. The respiratory distress syndrome of the newborn. Immaturity versus prematurity. Arch Dis Child 1965;40:62-5.

4 Dawkins M, MacGregor WG, eds. Gestational age, size and maturity. Clinics in Developmental Medicine No 19. London: Heinemann, 1965 .

5 Amiel-Tison C. Neurological evaluation of the maturity of newborn infants. Arch Dis Child 1968;43:89-93.

6 Dubowitz LMS, Dubowitz D, Goldberg C. Clinical assessment of gestational age in the newborn infant. 7 Pediatr
mentions ment of gestatior

7 Robinson RJ. Assessment of gestational age by neurological examination. Arch Dis Child 1966:41:437-47.

8 Ballard JL, Novak KK, Driver MA. A simplified score of fetal maturation of newly born infants. $\mathcal{F}$ Pediatr 1979;95:769-74

9 Parkin JM, Hey EN, Clowes JS. Rapid assessment of gestational age at birth. Arch Dis Child 1976;51:259-63.

10 Ballard JL, Khoury JC, Wedig K, Wang L, Eilers-Walsman BL, Lipp R. New Ballard score, expanded to include extremely premature infants. F Pediatr 1991;119:417-23.

11 The NNNI Trial Group. A randomised trial comparing the effect of prophylactic early fresh frozen plasma or gelatin 1996;348:229-32.

12 Thomson AM, Barron SL. A standard maternity information system. In: Chalmers I, McIwaine G, eds. Perinatal audit and surveillance. London: Royal College of Obstetricians and Gynaecologists, 1980:79-92.

13 Barron SL, Macfarlane AJ. Collection and use of routine maternity data. Baillière's Clin Obstet Gynaecol 1990;4:68197.
14 Vogt H, Haneberg B, Finne PH, Stensberg A. Clinical assessment of gestational age in the newborn infant. An 1981;70:669-72.

15 American Academy of Pediatrics Committee on Fetus and Newborn. Nomenclature for duration of gestation, birthweight and intrauterine growth. Pediatrics 1967;39:935-9.

16 European Association of Perinatal Medicine. Working party to discuss nomenclature based on gestational age and birthweight. Arch Dis Child 1970;45:730.

17 Usher RH, Allen AC, McClean FH. Risk of respiratory distress syndrome related to gestational age, route of delivery, 55 .

18 Morrison JJ, Rennie JM, Milton PJ. Neonatal respiratory morbidity and mode of delivery at term: influence of timing of elective caesarean section. Br 7 Obstet Gynaecol ing of elective caes

19 Madar J, Richmond S. Respiratory distress syndrome in babies born a term. Early Hum Devel 1997;49: (in press).

20 Casaer P, Daniels H, Devlieger H, De Cock P, Eggermont E. Feeding behaviour in preterm neonates. Early Hum Devel 1982:7:331-46.

21 Tin W, Wariyar W, Hey E. Changing prognosis for babies of less than 28 weeks' gestation in the north of England between 1983 and 1994. BM7 1997;314:107-11.

22 Spinnato JA, Sibal BM, Shaver DC, Anderson GD. Inaccuracy of Dubowitz gestational age in low birth weight racy of Dubowitz gestational age in

23 Shukla H, Atakent YS, Ferrara A, Topsis J, Antoine C. Postnatal overestimation of gestational age in preterm infants. Am $\mathcal{F}$ Dis Child 1987;141:1106-7.

24 Sanders M, Allen M, Alexander GR, et al. Gestational age assessment in preterm neonates weighing less than 1500 grams. Pediatrics 1991;88:542-6.

25 Alexander GR, de Caunes F, Helsey TC, Tompkins ME, Allen $M$. Validity of postnatal assessments of gestational age : comparison of the method of Ballard et al and early ultrasonography. Am f Obstet Gynecol 1992;166:891-5.

26 Constantine NA, Kraemer HC, Kendall-Tackett KA Bennett FC, Tyson JE, Gross RT. Use of physical and neurological observations in assessment of gestational age neurological observations in assessment of gestationa
in low birth weight infants. $\mathcal{7}$ Pediatr 1987;110:921-8.

27 Stephens-Simon C, Cullinan J, Stinson S, McAnarley ER Effects of race on the validity of clinical estimates of gestational age. F Pediatr 1989;115:1000-2.

28 Gagliardi L, Scimone F, DelPrete A, et al. Precision of gestational age assessment in the neonate. Acta Paediatr 1992; 81:95-9.

29 Donovan EF, Eherkranz RA, Tyson JE, Wright LE, Verter JJ. Inaccuracy of Ballard scores in estimating gestational age (GA) of 24-27 weeks infants of women with known menstrual history. Pediatr Res 1996; 39:206A.

30 Serfontein GL, Jaroszewicz AM. Estimation of gestational age at birth. Comparison of two methods. Arch Dis Child 1978; 53:509-11.

31 Gandy GM. Examination of the neonate including gestational assessment. In: Roberton NRC ed. Textbook of Neonatology. 2nd edn. Edinburgh: Churchill Livingstone, 1992.

32 Gardosi J, Mul T, Mongelli M, Wilcox M. Ultrasound dating and birth weight at term. Lancet 1994;308:1635.

33 Patterson CC, Halliday HL. Prediction of outcome shortly after delivery for the very low birthweight $(<1500 \mathrm{~g})$ infant. Paediatr Perinat Epidemiol 1988;2:221-8.

34 The International Neonatal Network. The CRIB (clinical risk index for babies) score: a tool for assessing initial risk of death and comparing the performance of neonatal intensive care units. Lancet 1993; 342:193-8.

35 Newens AJ, Jarvis SN, George R. How to gain weight by looking up. BMF 1992;305:1576-7. 